\title{
Les cinémathèques-musées au cœur de la pa- trimonialisation du cinéma en France (1936-1968)
}

\author{
Stéphanie-Emmanuelle Louis'
}

\begin{abstract}
Resumo
O museu tem funcionado como um mecanismo essencial para a patrimonialização do cinema na França. Produto da cinefilia do entreguerras, a Cinémathèque Française trabalhou desde a sua criação em 1936 para a assimilação da cinemateca a um museu de arte. $\mathrm{Na}$ ausência de um dispositivo estatal eficaz, ela se encarregou também da conservação de filmes em âmbito nacional até a criação de um serviço especializado junto ao CNC pós 1968. No processo de formalização e de organização das instituições patrimoniais na França, a Cinemateca-Museu foi percebida tanto como modelo como contra-modelo. Ela contribuiu para a conexão das noções de patrimônio e museu no campo do cinema.
\end{abstract}

\section{Palavras-chave}

Patrimônio cinematográfico; Cinematecas ; Museus de Cinema; Cinefilia

\begin{abstract}
Résumé
Le musée s'est avéré un rouage essentiel de la patrimonialisation du cinéma en France. Produit de la cinéphilie d'entre-deux-guerres, la Cinémathèque française a travaillé, depuis sa création en 1936, à l'assimilation de la cinémathèque à un musée d'art. Faute de dispositif étatique efficace, elle a parallèlement été préposée à la conservation des films au niveau national jusqu'à la création d'un service spécifique au CNC après 1968. Dans la formalisation et l'organisation des institutions patrimoniales en France, la cinémathèque-musée interviendra tant comme modèle que contre-modèle. Elle a contribué à connecter les notions de patrimoine et de musée en matière de cinéma.

\section{Mots-clés}

Patrimoine cinématographique; Cinémathèques; Musées du cinéma; Cinéphilie
\end{abstract}

Dans le cadre de cette réflexion sur les rapports entre patrimoine, cinéma et musée, nous montrerons comment le musée s'est avéré un rouage essentiel de la patrimonialisation du cinéma en France. Produit de la cinéphilie d'entre-deux-guerres, la Cinémathèque française a travaillé, depuis sa création en 1936, à l'assimilation de la cinémathèque à un musée d'art. Faute de dispositif étatique efficace, elle a parallèlement été préposée à la conservation des films au niveau national jusqu'à la création d'un service spécifique au Centre national de la cinématographie (CNC) après 1968. Dans la formalisation et l'organisation des institutions patrimoniales en France, la cinémathèque-musée interviendra tant comme modèle que contre-modèle. Mais avant d'étudier plus en détail cette trajectoire française, donnons quelques éléments de cadrage.

L'historien Dominique Poulot définit la patrimonialisation comme le processus d'« élaboration d'un cadre ou d'un instrument qui met l'accent sur les formes impersonnelles de conservation et de transmission de propriétés jadis personnelles. » (Poulot, 1992 :138) Concernant le cinéma, ces formes impersonnelles de conservation et de transmission peuvent être des musées du

I Membre du Centre Jean Mabillon-Ecole nationale des chartes-PSL Research University ; chercheuse invitée à l'Institut d'histoire du temps présent (IHTP-CNRS) ; louis.stefany@gmail.com 
cinéma, archives ou bibliothèques du film, le dépôt légal, ou encore les écrits historiographiques et manifestations commémoratives.

Différents travaux montrent qu'en France les éléments du dispositif de patrimonialisation sont mis en place dès la première moitié du $X X e$ Siècle. Selon Christophe Gauthier, la Cinémathèque française, fondée en 1936, contribuerait à «la coalescence de pratiques mémorielles et historiques apparemment divergentes» (2007 : 467). Au-delà, elle concrétise la mise en œuvre de la «cinémathèque moderne». II s'agit, selon Raymond Borde, historien et cinémathécaire, d'une cinémathèque distincte «des collections utilitaires à but pédagogique, juridique ou guerrier» (Borde, $1983: 75)$.

Afin d'éclairer le rôle déterminant joué par les cinémathèques dans la définition d'une politique patrimoniale spécifique au cinéma en France, nous évoquerons d'abord le passage à la cinémathèque moderne et la manière dont la Cinémathèque française a contribué à connecter les notions de patrimoine cinématographique et de musée du cinéma. Nous verrons ensuite comment le modèle de la cinémathèque-musée a caractérisé le processus de patrimonialisation en France, agissant comme levier dans son évolution. Enfin, nous envisagerons les interactions ayant placé les cinémathèques-musées dans une position de déclencheur d'une politique étatique dédiée avec la création du Service des archives du film du Centre national de la cinématographie.

\section{La cinémathèque, intermédiaire entre patrimoine et musée du ci- néma}

La presse française sur la première moitié du $X X$ siècle $^{2}$ laisse penser que les notions de patrimoine cinématographique, musée du cinéma et cinémathèque sont déconnectées avant que la Cinémathèque française devienne le moteur d'un réagencement décisif pour le processus de patrimonialisation au niveau national. C'est en fusionnant ces éléments qu'elle contribue à configurer un espace nouveau du monde cinématographique, dédié à son patrimoine.

- Des films que l'on possède et aux œuvres remarquables

Initialement, la presse qualifie de «patrimoine cinématographique» un ensemble de biens possédés et pas spécialement ce qui pourrait se transmettre aux générations futures comme le laissent penser plusieurs occurrences. En 1913, une annonce publicitaire signale que le programme d'actualités Sous la mitraille: pages sanglantes d'une conquête «appartient au patrimoine cinématographique de M. L.Aubert» (Comoedia, I913 : p.4 ; Le Petit Parisien, 1913 : p.7). II a en effet été filmé par un de ses opérateurs. La notion s'étoffe ensuite pour désigner également un élément remarquable dans la production cinématographique et un enrichissement culturel. En 1922, Le Matin déclare que, par ses qualités de reconstitution, Théodora (Leopoldo Carlucci, Italie, 1921) vient «enrichir le patrimoine cinématographique d'un document saisissant et précieux sur l'antique histoire.» («Théodora », Le Matin, I922 : p.5). De même pour l'admirable adap-

2 Plusieurs procédures ont été croisées : la consultation des archives de presse de la Cinémathèque française et celle des recueils de presse du département des Arts du spectacle de la Bibliothèque nationale de France, auxquelles se sont ajoutées des recherches thématiques et autour de personnalités-clés dans la base de données Calindex (https://calindex.eu/), dans la base de presse numérisée « Retronews » (https:// www.retronews.fr/). 
tation du Jocaste d'Anatole France par Gaston Ravel (France, 1925), qui gratifiera le «patrimoine cinématographique français d'une œuvre qui comptera parmi les meilleures de l'année» (« Jocaste », Le Matin, 1925 : p.4). Dans cette veine, en 1933, le quotidien culturel Comoedia affirme que Jean Epstein « [...] de Cœur fidèle jusqu'à L'Or des Mers a enrichi notre patrimoine cinématographique national d'œuvres riches de technique et d'intelligence » (Hombourger, 1933 : p.6).

- Les films du passé : un patrimoine à rendre visible

Des biens d'un individu à ceux de la nation, le spectre s'est élargi. De plus, les aspects temporels inhérents à la notion de patrimoine ont été déployés. D'abord capital présent, le patrimoine est devenu le vecteur de ce moment de la production cinématographique pour le futur et finalement manière d'élaborer un rapport au passé.

En effet, l'article sus-cité de Comoedia évoque «une vivante soirée anthologique consacrée à l'un des maîtres du cinéma français»; un «festival Jean Epstein», composé par «[...] quelques jeunes [...] qui ont encore le regret du passé à une époque où on a coutume de le renier, quelques jeunes qui connaissent le cinéma et qui l'aiment [...]» (Hombourger, 1933 : p.6). Organisée par Daniel Maybon et Marcel Alexis ${ }^{3}$, la soirée a été introduite par la conférence «Le cinéma et la vie» de Frédéric Lefèvre, rédacteur en chef des Nouvelles littéraires. Le programme comprend des extraits de La glace à trois faces (1927), Mor-Vran (I930), Finis Terrae (I93I), L'Or des mers (I93I) et L'homme à l'hispano (1932), et en intégralité : Le Cor (I93I), Le Vieux Chaland (1932). En fin de séance: «une date de l'histoire du cinéma» («La Vie des Lettres», Les Nouvelles littéraires, 1933); Cœur fidèle (1923) est présenté par Gina Manès, son interprète principale (Avronsart, 1933 : p.4) 4 .

Cet exemple met en évidence la pratique d'élaboration d'une généalogie artistique. Car ce sont ici de jeunes cinéphiles qui s'emparent du passé de l'art cinématographique pour en désigner une figure tutélaire. Ce phénomène caractéristique du processus de patrimonialisation, est désigné dans l'approche communicationnelle de Jean Davallon comme la mise en œuvre d'une filiation inversée (2006:27). C'est afin de répondre à ce désir cinéphile que seront créés le ciné-club «Cercle du Cinéma» et la Cinémathèque française, en 1935 et 1936. Car à cet égard, la Cinémathèque nationale inaugurée le 10 janvier 1933, sous l'égide du Secrétariat aux Beaux-Arts, ne donne pas satisfaction ${ }^{5}$.

Cependant, l'idée que les films anciens remarquables de l'art cinématographique forment un patrimoine et celle de les rendre accessibles progressent. En 1935, Paul Reboux, de la Commission de la Cinématographie pour l'Exposition internationale de 1937, évoque un projet de salle avec un programme en rotation sur 3 à 4 jours, composé d' «une vingtaine de films français, sélectionnés parmi les chefs-d'œuvre de ces dix dernières années» et qui per-

3 Elle se tint le ler juin 1933, 2 Ih, salle Adyar, 35 avenue Rapp à Paris. D'autres soirées de ce type eurent lieu en 1933. Daniel Maybon intégra le Guide du Concert pour y tenir la rubrique cinématographique.

4 Remarquons avec Christophe Gauthier que la projection d'extraits serait une formule commune dans la cinéphilie d'entre-deux-guerres. Outre son utilisation par Ricciotto Canudo au Salon d'Automne entre 1921 et 1923, elle aurait été mise en œuvre par Antoine lors de la séance inaugurale du ciné-club de France le 12 juin 1920 (2007 : 194).

5 Sur l'échec de la Cinémathèque nationale, l'histoire du Cercle du cinéma et de la Cinémathèque française, voir Laurent Mannoni (2006). 
mettrait « en quelques heures de prendre contact avec l'ensemble de notre patrimoine cinématographique»; l'objectif étant de promouvoir la qualité de la production française. II parle alors d'un «Conservatoire de cinéma» (Casanova, 1935: p.9).

- La connexion patrimoine-cinémathèque-musée après la Seconde guerre mondiale

Bien que la réflexion existe sur ce que sont les «cinémathèques» ou pourrait être un «musée du cinéma» auparavant, il semble que les rapprochements explicites dans le discours entre «patrimoine», «musée» et «cinémathèque» n'interviennent qu'après la Seconde guerre mondiale. Parallèlement, de 1945 à 1950, l'usage de la terminologie «patrimoine cinématographique» passe des propos de presse à une appropriation institutionnelle par la Cinémathèque française. Les questions de préservation deviendront dès lors indissociables des activités des cinémathèques modernes dans le débat public.

En août 1945, l'Ecran français publie un article de Jean-George Auriol', intitulé Les Films patrimoine artistique. Il y est question de la Cinémathèque française et de la remarquable activité d'Henri Langlois ${ }^{7}$ depuis les années 1930, notamment sous l'Occupation ${ }^{8}$, pour sauver les films anciens (Jameson, 1945: p. 12-13). Auriol décrit la complexité de l'organisation existante, et à développer, regroupant sous le titre de «patrimoine» et la bannière de la Cinémathèque française l'ensemble des fonctions patrimoniales qui étaient débattues jusqu'alors essentiellement de manière séparée. La «bibliothèque des films» pour ce qui concerne la documentation sur la production cinématographique; à savoir: bibliothèque ${ }^{9}$, service de documentation photographique, service de presse sur l'art, l'histoire et les métiers du cinéma, et aussi de répertoire filmographique. Les «archives du film» pour la collecte des «copies égarées, oubliées». Le «dépôt obligatoire» pour «les films nouveaux», sur modèle du dépôt légal. Le «musée du cinéma» avec notamment des expositions de collections non-film, concluant que celles-ci feront «une merveilleuse propagande à l'esprit d'invention et au goût français.» (Amable Jameson, 1945). Bien au-delà, elles seront l'un des fers de lance de la promotion d'un modèle de valorisation patrimonial tout aussi singulier dans la communauté internationale des archives du film, qu'inspirant pour les cinéphiles français et étrangers.

La cristallisation du rapport entre patrimoine, cinémathèque et musée du cinéma se poursuit au cours des années suivantes autour de la Cinémathèque française. Fin 1947, Jean Grémillon, qui préside alors l'association, pose, dans un texte destiné aux partenaires ou financeurs, la cinémathèque comme une des organismes devant contribuer à enrichir le «patrimoine culturel national.» (Grémillon, 2010 : 247-248) En 1950 la notion de «patrimoine cinématographi-

6 II publie alors sous le pseudonyme d'Amable Jameson

7 La Cinémathèque française a été fondée par Henri Langlois, Georges Franju, Paul-Auguste Harlé et Jean Mitry en 1936. Mais après la Seconde Guerre mondiale, la personnalité de Langlois devient prédominante par rapport aux autres dans le récit de la naissance de l'association.

8 Durant la Seconde Guerre mondiale, suite à l'Armistice du 22 juin 1940 le pays fut divisé par une ligne de démarcation. La zone Nord de la France, où se situait Paris et la Cinémathèque française, a été occupée par l'Armée du Ille Reich. Au sud subsistait une « zone libre », où put notamment se cacher l'historienne d'origine juive Lotte Eisner. Le territoire continental fut libéré à partir de l'été 1944 (Libération). Paris sera libérée le 25 août 1944 et les activités de la cinémathèque reprendront progressivement en fin d'année. 9 Un projet avait déjà été mis en œuvre par les époux Moussinac dans les années 1920.Voir : Léon Moussinac, « Sur la création d'une bibliothèque du cinématographe et son organisation », Monde, 27/01/1929. 
que national» réapparaîtrait publiquement présentée dans un article de Langlois, intégré au chapitre sur les «Nouvelles formes de conservation de la pensée» d'un numéro spécial d'Education nationale consacré aux Bibliothèques.

Sur la préservation des films, il déclare : «Tandis que les stocks de la Cinémathèque française, par un enrichissement constant, tendent à ne plus être une sélection des grands films internationaux, mais à grouper la totalité du patrimoine cinématographique national dans un blockhaus vaste, où les films pourront à la fois être stockés et conservés grâce à des aménagements et des travaux qui doivent en faire un des plus grands et des meilleurs blockhaus.» (Langlois, 1950: p.44). Qu'entend exactement Langlois par «patrimoine cinématographique national»? Est-ce, à l'instar des discours des années 1920 et 1930, un ensemble de films français remarquables? Est-ce une projection de «cette cinémathèque idéale qui conserverait toute la production nationale» (Langlois, 1949 : p.88) que Langlois appelait de ses vœux en 1949? Est-ce, plus concrètement, un écho à ce que le Centre national de la cinématographie qualifie de «patrimoine film» (CNC, 1949 : p.2) à la fin des années 1940; désignant ainsi les films ayant reçu une aide étatique à la production?

L'importance de ce texte est multiple. D'abord, il associe publiquement, via la presse, la cinémathèque et le musée au devenir patrimonial du cinéma. Ensuite, il situe la Cinémathèque française parmi ses pairs et la signale comme un organisme indépendant agissant pour le compte de l'Etat pour préserver le cinéma. Enfin, il décrit un organisme de valorisation original. Langlois évoque les activités de collecte et de projection de films internationaux de la Cinémathèque française, contribuant ainsi à la diffusion de la culture cinématographique, mais aussi la «charge du Musée du Cinéma» et la «bibliothèque spécialisée parmi les plus éclectiques sur le plan international» (Langlois, 1949 : p.88), qui associe livres, revues, quotidiens, affiches, scenarii, plaquettes publicitaires, manuscrits, maquettes de décors et de témoignages rassemblés via la Commission de recherches historiques. En somme, Langlois promeut un modèle de valorisation patrimoniale.

\section{La cinémathèque-musée, un modèle innovant de patrimonialisa- tion en France}

En I 949 Langlois déclarait dans la revue l'Amour de l'art:

Au mot de cinémathèque, on évoque immédiatement un immense entrepôt fermé à double tour où s'accumulent des milliers de bobines, une institution impersonnelle dont la seule raison d'être est de collectionner d'office le plus grand nombre de films. [...] Qu'on ne vienne pas nous dire que Cinémathèque et Musée du cinéma sont deux choses différentes [...]. (Langlois, 1949).

Au cours des décennies suivantes, la Cinémathèque française s'applique à mettre en œuvre cette fusion.Vers 196I, Langlois défendra un projet de «Cinémathèque-Musée» devant la Commission au Plan chargée de financer les grands travaux (Mannoni, 2006: 32I-322). De plus, différents groupes de cinéphiles, notamment en France, s'approprieront le modèle proposé par la Cinémathèque française, inscrivant alors le processus de patrimonialisation dans une géographie décentralisée. 
- Le musée du cinéma comme rétrospective des techniques

Evoquons d'abord brièvement l'évolution de la notion de «musée du cinéma» dans la première moitié du XXe Siècle. II s'agit premièrement d'un ensemble d'appareils apparenté donc à un musée des techniques (Gauthier, 2007: I53-162 $)^{10}$. A cet égard, quand Comoedia appelle, suite à l'exposition l'Art dans le cinéma français tenue en 1924 au Musée Galliera, à créer «le 'musée du Cinéma' qui, dès aujourd'hui et sans retard, s'impose»: il s'agit de donner une visibilité pérenne à «la partie rétrospective où sont exposés les appareils des précurseurs de cette industrie qu'auréole déjà un si grand prestige esthétique.» (Danvers, 1924: p.I). Le «musée du cinéma» inauguré en 1927, sous la forme d'une section cinématographique au Conservatoire national des arts et métiers (CNAM) répond à ce besoin" .

En 1929, Comoedia tente de mobiliser son lectorat autour d'un modèle alternatif de «musée du Cinéma français», s'inspirant d'une initiative américaine regroupant «[...] des objets ayant appartenu à nos vedettes, des costumes qu'elles ont portés dans telle création sensationnelle, des meubles, des maquettes de décors» (Lebreton, 1929: p.6). Toutefois la proposition semble rester lettre morte et en 1934, la presse relaie toujours, autour du projet de cinémathèque nationale, que «le musée du Cinéma et de la photographie» sera une exposition d'appareils (Chataigner, 1934 :p.6). L'Exposition internationale de 1937 et les débats qu'elle suscite constitue un bon observatoire de la transformation qui adviendra.

- La mutation du musée au milieu des années 1930

Les initiatives étrangères stimulent la réflexion. En janvier 1936, un journaliste de Comoedia conclut de sa visite aux studios de Neubabelsberg et de Tempelhof :

Pourquoi ne pas avouer que cet exemple mériterait d'être suivi. [...] Chez Lumière, chez Gaumont, chez Pathé, chez Debrie, ailleurs, il y a des machines et des documents. Des particuliers même se feraient un plaisir d'ouvrir des dossiers, de donner des livres, des lettres, des manuscrits. Les bonnes volontés ne manqueraient pas et l'invention du cinéma est encore assez proche de nous pour que l'inauguration d'un Musée permette un regroupement de toutes les valeurs présentes et passées de nos techniciens et de nos artistes. (Liausu , $1936:$ p.6)

Quelques mois plus tard, alors qu'une circulaire ministérielle institue une Commission du cinéma d'enseignement au ministère de l'Education nationale, dotée d'une sous-commission ayant pour but de constituer un musée du cinéma, Paris-Soir interpelle son lectorat: «[...] que va-t-on y mettre? Des appareils, bien sûr. Mais si c'est fort intéressant, ce n'est pas très original. Que peut-on faire pour rendre le musée du cinéma intéressant, instructif, attrayant et même amusant pour assurer son succès?» (Thierry, 1936: p.6) L'enquête sus-

10 Voir la thèse de Christophe Gauthier précédemment citée.

II A ce sujet, voir la revue de presse de la Bibliothèque nationale de France : Bibliothèques, Filmathèques et Archives du Cinéma : recueil d'articles 8-RK-582 (I) 
cite l'intérêt chez les lecteurs et le journal publie notamment les suggestions de Melle Berques qui souhaite que le Musée du Cinéma comprenne trois sections:

I - Fabrication des appareils et des films (des procédés anciens aux plus modernes) ;

2 - Fabrication d'un film (maquettes, studios, décors, truquages, etc.) 3 - Le film et la projection (cinécothèque, cinémathèque, salle de projection).

Des conférences auraient lieu chaque semaine, et des films de professionnels seraient projetés, les spectateurs étant invités à discuter la valeur des œuvres présentées. (Thierry, 1936b: p.8)

- Le positionnement de la Cinémathèque française

En septembre, le débat est relancé dans les colonnes de la presse sous la plume inattendue de Léo Deglesne ${ }^{12}$. Dans le bimensuel la Griffe, il publie un texte programmatique intitulé «Pour un Musée du cinéma», où il invite à «une organisation moderne, une présentation nouvelle. [...] afin de réaliser pour la cinématographie un musée aussi vivant que cet art lui-même.» (Deglesne, 1936: 8). Il envisage un découpage en trois sections. D'abord, autour de la fabrication de l'image: un «département industriel» avec les appareils, mais aussi les activités des studios et laboratoires. Ensuite, un «département commercial», centré sur l'exploitation: sortie du film, aménagement et architecture des salles anciennes et contemporaines, publicité cinématographique, mais aussi chiffres de fréquentation sont autant d'aspects inclus au programme de Deglesne. Enfin un «département artistique», sur «les auteurs, les réalisateurs (metteurs en scène), les compositeurs, les artistes; les paysages, les décors, les costumes, les accessoires. [dont] Une grande place serait réservée à une 'cinémathèque'» (Deglesne, 1936:8). Deglesne envisage celle-ci comme une collection de films qui pourrait donner lieu à des échanges avec l'étranger. II invite à générer des ressources financières pour le musée grâce à des projections de classiques, mais aussi des cours avec films scientifiques, commémorations appuyées sur des documentaires. Sans compter la mise en place d'un système de location. II intègre aussi une bibliothèque au projet de musée. Si certains dénigrent le programme de Deglesne (d'Herbeumont, 1936 : 176), d'autres font bon accueil à ses suggestions. Elles seront en grande partie reprises par le quotidien culturel Comoedia afin « que cette industrie nouvelle - à laquelle collaborent toutes les techniques et tous les arts - possèdent [sic] son 'centre' historique.» (Emile-Bayard, 1936)

En octobre, Langlois réagit au nom de la Cinémathèque française, association qui a été déclarée le 9 septembre 1936 dans le but de préserver à la fois des objets film et non-film et de favoriser leur diffusion (Mannoni, 2006: 46-47). Quant à la faisabilité du modèle proposé par Deglesne, Langlois signale que le «département industriel» pourrait être constitué à partir du fonds du CNAM. II poursuit :

\footnotetext{
D'autre part, je ne vous apprends pas la création de la Cinémathèque française par l'association des membres les plus importants de la corporation (metteurs en scène, producteurs, distributeurs, dé-
}

12 Léo Deglesne fut auteur de théâtre, poète et chansonnier, il fut aussi représentant commercial entre les années 1910 et la fin des années 1930. II n'apparaît pas comme un contributeur régulier de la Griffe; périodique bimensuel provenant de la fusion, en 1934, entre la Griffe cinématographique et la Griffe financière, politique, théâtrale et littéraire, qui paraissaient alternativement depuis 1920. 
corateurs, acteurs, opérateurs) dans le but de recueillir, d'entretenir et de permettre la vision des films hors exploitation ${ }^{13}$.

Comme vous le savez, la Cinémathèque française, qui a pu recueillir déjà un assez grand nombre de documents ayant trait au cinéma (maquettes, affiches, manuscrits, photos, etc.), a réuni en outre, à quelques exceptions près, tous les films caractéristiques du cinéma français. [...] plus des bandes françaises et des films français envoyés à l'étranger grâce aux échanges établis avec les cinémathèques d'autres nations. (Langlois, 1936: p.4)

II signale que des programmes seront prochainement tenus à disposition des universités, écoles et associations et affirme que «la Cinémathèque française peut parfaitement assumer le rôle de département artistique.» II poursuit :

\begin{abstract}
Pour que le Musée ou la Cité du Cinéma soit une réalité, il ne reste plus donc qu'à organiser le département commercial qui est prévu et à trouver l'argent nécessaire à son entretien et à son fonctionnement.

La France sera dotée alors d'un établissement unique en Europe, dépassant tout ce que nos voisins ont tenté dans ce sens, et le seul qui puisse rivaliser avec The Film Library de New-York ${ }^{14}$, qui, comme vous le savez, n'a pu être que grâce à la fortune des Rockefeller. (Langlois, 1936: p.4)
\end{abstract}

La presse si elle considère que l'Etat devrait appuyer le projet, peut-être échaudée par la stagnation de la Cinémathèque nationale, encourage aussi «l'initiative privée» et la corporation à investir dans ce domaine (Emile-Bayard, 1936; Laspeyres, 1936: p.4). La Cinémathèque française s'impose ensuite comme leader français de cette conquête muséale. En 1938, elle participe à la création de la Fédération internationale des archives du film (FIAF); en 1939, elle récupère les prérogatives de la Cinémathèque nationale (Mannoni, 2006: 62-75) A la Libération, elle se pose comme un incontournable centre français des pratiques historiques et mémorielles du cinéma.

\title{
- Renouveler la représentation de la cinémathèque
}

Cependant, il reste encore à transformer la représentation de ce qu'est une cinémathèque, comme l'a écrit Langlois dans l'Amour de l'art. Tel est bien le projet de la Cinémathèque française selon les écrits de Jean Grémillon, lorsqu'il évoque la naissance d'une «véritable cinémathèque.» (Grémillon, 2010: 246) Jusqu'aux années 1930, les cinémathèques existant en France étaient essentiellement des centrales de prêt facilitant l'organisation de projections, surtout à des fins d'enseignement ou de propagande, telles les cinémathèques des ministères ou des offices de cinéma éducateur par exemple. Elles préservaient les films dans la mesure où ils pouvaient encore alimenter les séances, sans s'interroger sur une conservation pour une histoire à long terme. La Cinémathèque française souhaite mettre en œuvre une conservation au sens plein du terme, et cela suppose des moyens importants, comme l'a malheureusement montré le cas de la Cinémathèque nationale. Au-delà de la conservation, sa mission de diffusion est essentielle:

13 C'est en effet cet aspect qui a été le plus mis en avant dans les deux annonces parues dans la Cinématographie française, $n^{\circ} 934,26 / 09 / 1936$ et $n^{\circ} 932,12 / 09 / 1936$.

14 II s'agit de la Film Library du Museum of Modern Art qui constitue une très forte inspiration pour Langlois. 
Dans la mesure où l'on souhaite que la Cinémathèque soit autre chose qu'un stock de bobines et que le caractère d'œuvre des films retrouvés soit également préservé, il devient nécessaire d'assurer régulièrement des projections à des fins non commerciale naturellement, des films entreposés. (Grémillon, 2010:248)

Elle a aussi vocation à organiser des manifestations, des expositions, des cours, appuyés et complétés par des publications. Et, nuance fondamentale quant aux organismes qui l'ont précédée, elle doit servir la valorisation artistique du cinéma: «La fonction principale d'une cinémathèque est de donner en fait aux films produits, le caractère d'œuvres d'art que l'organisation industrielle et commerciale contemporaine de la production leur arrache.» (Grémillon, 2010:252) Loin d'être uniquement une bibliothèque - modèle systématiquement évoqué dans la presse pour marquer l'ambition de collecter le dépôt légal ${ }^{15}$ - la cinémathèque moderne serait donc aussi un musée d'art.

Les activités de transmission et de participation à l'écriture de l'histoire du cinéma nourriront la controverse autour de la Cinémathèque française et, plus largement, de la préservation du patrimoine cinématographique.Autour de sa création, la presse a signalé qu'elle mettrait « à la disposition des universités, écoles, associations qui lui en feront la demande une suite de programmes retraçant l'histoire du cinéma français ॥ (Laspeyres, 1936: p.4), le fait que ceci ne se concrétise que très partiellement et soit finalement abandonné dès les années 1950 sera facteur de polémique dans une France d'après-guerre qui connaît une véritable expansion cinéphile sous la houlette des ciné-clubs ${ }^{16}$. De plus, les expositions film et non-film de Langlois ne feront jamais oublier «les rouleaux de films [...] soigneusement rangés» (Dartois, 1928: p.8 dans les armoires, la «jeune ouvrière occupée dans son atelier à 'retaper' les pellicules» (MP, 1932, p.6) des cinémathèques qui ont précédé; encore moins les pratiques d'organismes étrangers contemporains membres de la FIAF.

La Cinémathèque française est néanmoins soutenue par l'Etat depuis octobre 1943. Le 21 juin de la même année, le dépôt légal a été institué sans pourtant que soit publié de décret d'application. Finalement, le soutien étatique maintenu après la Libération permettra à la Cinémathèque française de pallier imparfaitement cette carence alors que l'Etat définira, dans des interactions parfois tumultueuses, un positionnement autonome en matière de valorisation patrimoniale du cinéma.

\section{La Cinémathèque française-Musée du cinéma, du levier institu- tionnel au déclencheur politique}

Malgré les critiques qui lui ont été adressées, la Cinémathèque française a inspiré les cinéphiles de son temps et son modèle de cinémathèque-musée a été réapproprié en France comme à l'étranger. Pour ce qui est du cas fran-

I5 Dispositif légal institué en France en 1537 et permettant la collecte et la conservation de collections de référence. Initialement appliqué au livre, il s'est successivement étendu jusqu'à celui de l'internet, en passant par l'affiche, la photographie ou le disque. Les documents concernés doivent être obligatoirement déposés par leur éditeur, imprimeur, producteur ou importateur auprès des institutions habilitées $(\mathrm{Bi}$ bliothèque nationale de France, Institut national de l'Audiovisuel, Centre national du cinéma et de l'image animée).

16 Au sujet de la querelle avec la Fédération française de ciné-clubs, voir Laurent Mannoni (2006 : 166 174). Sur le mouvement ciné-club, voir Léo Souillès-Débats (2017). Sur les controverses autour des activités de la Cinémathèque française entre 1944 et 1968, voir Louis (2013). 
çais, l'apparition de ses avatars met progressivement en évidence les limites de l'étalon: difficultés à irriguer convenablement tout le territoire, choix de ne donner accès qu'à certains types de films. II en ira de même avec la décision de créer un service de archives du film au CNC, entérinant l'incapacité de la Cinémathèque française à assurer la conservation pérenne et l'inventaire des stocks de films. Ce faisant, l'espace de patrimonialisation que celle-ci a contribué à faire apparaître se reconfigure progressivement.

- La question de l'accessibilité des collections

Cruciale, la question de l'accessibilité des films se pose sous différentes formes. Pour diffuser régulièrement ses films hors de Paris, des sections d'Amis de la Cinémathèque sont créées à partir du début des années 1950, suite à la rupture avec la Fédération française de ciné-clubs (FFCC) (Louis, 20I3). La correspondance avec Raymond Borde, animateur de la section toulousaine, montre les multiples difficultés liées à l'organisation des séances : envoi trop tardif de copies, absence de sous-titrage, changements inopinés de programme. Quant au Centre Régional de documentation pédagogique (CRDP) de Lyon, pourtant désireux et matériellement prêt à ouvrir une section amie, ses démarches resteront vaines (Louis, 20I4-I).

Outre la difficulté de répondre aux sollicitations des groupements cinéphiliques de province en quête de films anciens, il arrive aussi que la Cinémathèque française ignore les requêtes adressées par certains historiens. Entre novembre 1960 et février 196I, Borde demande par exemple le prêt de «trois ou quatre anciens Fritz Lang» ${ }^{17}$ afin de travailler à une étude avec Francis Courtade; finalement, il «s'arrangera pour voir ces films dans une Cinémathèque étrangère ${ }^{18}$.

Parfois aussi, la Cinémathèque française brille par son absence de collaboration à des projets historiographiques ambitieux. Dans le deuxième tome l'Histoire comparée du cinéma, Jacques Deslandes ${ }^{19}$ et Jacques Richard écrivent:

on ne saurait non plus parler d'un film en employant les méthodes qui ont servi à son lancement publicitaire - ou à celui d'une vedette ; et si l'obscurité est nécessaire à la projection d'une bande cinématographique, sa préservation et sa conservation ne sauraient cependant nécessiter les ténèbres du mystère. Une cinémathèque n'est point une grotte comme celle où Ali-Baba découvrit les richesses des quarante voleurs. C'est pourquoi il faut saluer l'initiative du British Film Institute à Londres qui, notamment en publiant l'inventaire détaillé des films, a fourni au chercheur un indispensable instrument de travail. (Deslandes, Richard, 1968: 7-8)

Concernant les documents non-film, ils orientent vers les collections

\footnotetext{
17 Lettre de Raymond Borde à Mary Meerson, 19/I I/1960, If.dact, La Cinémathèque de Toulouse, Correspondance de Raymond Borde, non coté et aussi Lettre de Raymond Borde à Mary Meerson, 26/01/196I, If.dact, et Lettre de Raymond Borde à Henri Langlois, 0I/02/196I, If.dact ; La Cinémathèque de Toulouse, Correspondance de Raymond Borde, non coté.

18 Lettre de Raymond Borde à Henri Langlois, 09/05/I96I, 3f.dact; La Cinémathèque de Toulouse, Correspondance de Raymond Borde, non coté.

19 Notons que Jacques Deslandes a écrit en 1961 dans le catalogue de l'Exposition commémorative du centenaire de Georges Méliès, organisée par la Cinémathèque française au Musée des arts décoratifs.
} 
du Cabinet des Estampes de la Bibliothèque Nationale ${ }^{20}$, le Fonds Rondel de la Bibliothèque de l'Arsenal dont «le catalogue manuscrit 'cinéma' [qui] permet à I'historien de s'orienter rapidement et utilement» (Deslandes, Richard, 1968: 767), précisant qu'il est accessible à tous. Les auteurs expriment d'ailleurs leur gratitude aux conservateurs et bibliothécaires. En outre, les crédits portés à la table des illustrations qui mentionnent, en sus des collections privées, les fonds publics de la $\mathrm{BN}$, de la Phonothèque nationale ou du CNAM.

Autour de cette question de l'accessibilité des collections film et non-film pour faire l'histoire du cinéma se forment des projets de cinémathèques-musées alternatifs. A Toulouse, des instances provisoires sont créées, rendant consultables les collections constituées localement, mais visant aussi à les protéger en cas de litige avec la Cinémathèque française. Un «groupement de recherches cinématographiques» avait été créé de manière informelle en octobre 195921. Il est officialisé en lien avec le Centre régional de documentation pédagogique de Toulouse, sous la forme d'une Commission d'études et de recherches cinématographiques fondée par un arrêté du Rectorat daté du 16 janvier 1960. Elle a pour objectif d'alimenter un dépôt de documents non-film en vue de publications pédagogiques ${ }^{22}$ (Icart, 1960: np) En 196, le Centre d'Histoire du cinéma ${ }^{23}$ couvre un champ plus large (Borde, 1990: 7). Après sa création en 1964, la Cinémathèque de Toulouse répondra notamment aux demandes de prêt d'organismes émergents comme le Comité de Fondation du Musée du Cinéma et de la Cinémathèque de Lyon. Créé en décembre $1965^{24}$, celui-ci réunit différents acteurs lyonnais: le Docteur Paul Génard, détenteur d'une collection d'appareils, et Charles Perrin, du CRDP, qui avait tenté d'installer une section d'Amis de la Cinémathèque française à Lyon et dont les anciens films muets de l'office du cinéma éducateur ${ }^{25}$ ont été déposés au stock des Amis de la Cinémathèque à Toulouse. Si ces deux initiatives montrent qu'une histoire générale du cinéma peut-être préservée, écrite et diffusée ailleurs qu'à Paris, la mise en œuvre d'un projet spécifique au cinéma d'animation à Annecy signale que toutes les pratiques cinématographiques et domaines d'intérêt historiographiques ne sont pas valorisés par la Cinémathèque française ${ }^{26}$ (Louis, 2014-2).

20 Issue des collections royales la Bibliothèque nationale a été créée suite à la Révolution française en 1789. Elle est devenue la Bibliothèque nationale de France suite au décret $n^{\circ} 94-3$ du 3 janvier 1994 portant création de la Bibliothèque nationale de France.

21 « Les programmes de février et de mars à la Cinémathèque française », sl, 05/02/1960. Cinémathèque de Toulouse, Presse, Boîte 1958-1987 ; La création est aussi annoncée dans le programme de la scetion des amis de la Cinémathèque, à Toulouse, du mois de novembre 1959. Cinémathèque de Toulouse, Programmes. 22 La Commission est en effet à l'origine de plusieurs publications entre 1960 et I964, qui permettent de bien marquer son existence institutionnelle (Icart, 1960; Pornon, 1961; Pornon 1964).

23 Outre le témoignage de Raymond Borde, et la mention explicite «Centre d'Histoire du cinéma » dans certaines entêtes de courriers à partir de 1962, nous ne disposons pas d'informations complémentaires à l'heure actuelle. Les courriers mentionnés font partie de la correspondance de Raymond Borde conservée à la Cinémathèque de Toulouse.

24 La création s'étale entre décembre 1965, où un arrêté municipal installe le Comité, et juillet 1966, où le statut associatif est adopté pour permettre le versement de subventions municipales. " Le Musée du cinéma de Lyon », Dissolution prononcée par l'Assemblée générale, 23/0I/200I, 7f.dact.

Archives Charles Perrin.

25 Apparus dans les années 1920, les offices du cinéma éducateurs étaient des organismes d'éducation par le cinéma, ils s'appliquaient à diffuser les valeurs républicaines et laïques notamment face aux salles des patronages religieux. A la fin des années 1940, ils beaucoup furent transformés en Offices régionaux des œuvres laïques d'enseignement par l'image et le son (OROLEIS).

26 Notons néanmoins que la Cinémathèque française avait organisé une exposition sur le Dessin animé entre novembre 1945 et 1946 avenue de Messine (Louis, $2013: 63$ ). 


\section{- Le problème d'une conservation rationnelle}

La difficile accessibilité des collections de la Cinémathèque française provient peut-être d'un manque de moyens, mais plus largement d'une stratégie de gestion consistant à privilégier les activités spectaculaires au traitement systématique des collections film. On tend plutôt à une conservation intellectuelle des œuvres grâce à leur visibilité, et à cette fin des opérations de restauration, de retirage peuvent être réalisées; l'inventaire et le stockage rigoureux ne sont pas des priorités. En 1949, le CNC a mis à disposition de la Cinémathèque française des bâtiments de l'ancien fort militaire de Bois d'Arcy en vue de classement et d'inventaire des fonds de films; ils ne sont pas adaptés à la conservation. Et, en 1954, alors que la Cinémathèque française a récupéré de nombreux films nitrate du fait de l'interdiction de ce support ${ }^{27}$, Langlois déclare au Conseil d'administration «un des rôles de la Cinémathèque étant de conserver les films - mais ce sont des choses très coûteuses et j'attendrai que la Cinémathèque soit très riche pour le faire ${ }^{28}$. Les professionnels du cinéma contestent de plus en plus la gestion de l'association. Leurs revendications trouvent parfois un écho auprès de l'administration de tutelle qui demande régulièrement des comptes sur les activités de conservation.

En 1959, l'incendie de la rue de Courcelles ${ }^{29}$ pointe la désorganisation de la Cinémathèque française pour la gestion de copies et la discrédite au sein de la FIAF $^{30}$. Cependant, l'arrivée d'André Malraux au Ministère de affaires culturelles, et le passage du cinéma dans le portefeuille de cette nouvelle administration, marque une trêve. La Cinémathèque française devient un élément de la politique culturelle nationale; ses moyens augmentent. Mais bientôt, le projet de nationalisation de l'association crispe les rapports avec l'Etat. Cependant, en 1963, une belle salle de projection est inaugurée au Palais de Chaillot, et assortie de quelques vitrines d'exposition dans le hall en attendant l'ouverture d'un vaste parcours permanent dans les galeries.

Depuis les années 1940, la cinémathèque française a été soumise à plusieurs vérifications de l'Inspection générale de finances, cependant, le rapport Heilbronner (avril 1965) est particulièrement critique (Mannoni, 2006 : 335). Pour mettre en place une gestion rationnelle, il recommande la nationalisation, l'amélioration des conditions de conservation et l'application du dépôt légal. De plus, rappelons avec Malraux, que la législation et la réglementation générales sur le classement et la conservation des monuments historiques, objets historiques et artistiques (loi du $31 / / 2 / 19 / 3$ ) ne comporte pas «de dispositions particulières au patrimoine cinématographique» et que «la plupart des appareils et documents dont il s'agit sont en la possession de la cinémathèque française ${ }^{3 !}$.

27 A partir de 195I en France, des mesures successives tendent à remplacer le film nitrate, hautement inflammable, par le film sur support acétate.

28 Cinémathèque française, Conseil d'administration, 06/05/I954, p. 17. Archives nationales, 19900289 : Culture, Centre National de la Cinématographie Française ; Archives de la direction générale. $X$ : Conservation ;Art.8I.

29 En 1944, la Cinémathèque française a ouvert ses premiers locaux avenue de Messine. Après son expulsion en 1955, elle s'installe rue de Courcelles avait d'emménager au Palais de Chaillot à partir de 1963. 30 La Cinémathèque française quittera d'ailleurs la FIAF au début des années 1960, pour y revenir en qualité d'observateur en 1983 puis de membre plein en I99I.

31 Journal Officiel de la République française, 23/10/1962 : réponse à la question écrite $n^{\circ} 2.969$ de M. George Lamousse. 
- La crise de l'inventaire et l'affirmation d'un projet d'Etat

Alors que la Cinémathèque française se concentre sur les activités spectaculaires, la communauté internationale s'interroge et cadre les pratiques de conservation. Une commission «Préservation» est créée à la FIAF en 196I, elle conçoit un manuel - The Preservation of Film - publié en trois langues, dont le français ${ }^{32}$, entre 1963 et 1967. A I'UNESCO, le Conseil international du cinéma et de la télévision confie une Etude sur la création de centres nationaux de catalogage de films et de programmes de télévision, à Jacques Ledoux, le conservateur de la Cinémathèque royale de Belgique. Il y met en évidence les conséquences regrettables de l'absence de catalogues de films centralisés. Parallèlement en France, l'heure est au dénombrement du patrimoine national: en mars 1964, une Commission nationale chargée de préparer l'établissement de l'inventaire général des monuments et des richesses artistiques de la France a, par exemple, été instituée par décret (Poirrier, 2003: 225) ${ }^{33}$. Dans ses souvenirs, André Holleaux, qui fut directeur de cabinet de Malraux puis directeur général du CNC, insiste sur le fait que «l'Inventaire général se voulait un répertoire scientifique» (Comité d'histoire du Ministère de la culture, 2004: I50). L'Inventaire général constituait, dans l'esprit de Malraux, l'un des outils de «la mémoire, mieux la substance de la France passée qui, pour lui comme pour les artistes, donnent des racines à la création et préfigurent le futur. II fallait donc d'abord constater, classer et ordonner dans un souci scientifique») ${ }^{34}$ (Comité d'histoire du Ministère de la culture, 2004: 150).

Dans ce contexte, le Centre National de la Cinématographie lance une initiative de fichier centralisé ${ }^{35}$ avec en publiant une Liste des films constituant le patrimoine cinématographique de l'Etat. Ce catalogue «édité spécialement à l'usage des différents Départements Ministériels, comprend l'ensemble des films venus compléter le Patrimoine Cinématographique de l'Etat, depuis le mois de mai 1965» (CNC, 1965). A terme, il doit aussi intégrer des mises à jour ainsi que les films inventoriés dans les ministères avant 1965. Ce nouvel outil entend servir à une large diffusion des films. Dès lors les tensions entre l'Etat et l'association se cristallisent autour de la question de l'inventaire. Si l'Etat s'applique à recenser les éléments de ses propres collections, il apparaît compréhensible qu'il attende la même chose de la Cinémathèque française, figure de proue, et largement financée, de sa politique patrimoniale.

Le 2 juillet 1965, Holleaux et Malraux créent une «Commission chargée de préparer le plan de conservation des films détenus par la Cinémathèque française» (Bulletin d'information du CNC, 1967: p.26). Le 7 octobre 1965 un arrêté du Ministère d'Etat chargé des Affaires culturelles définit les «Conditions d'utilisation des crédits destinés à couvrir les dépenses de tirage de copies de films détenus par la Cinémathèque française $\iota^{36}$, qui doivent répondre à un

32 Deslandes et Richard y font d'ailleurs référence dans leur ouvrage, en soulignant l'activité remarquable du British Film Institute (BFI) en la matière (1968:8).

33 Décret n64-203, Journal Officiel de la République française, 08/03// 964 (Poirrier, $2003: 225$ )

34 Ibid., p. 153

35 Cette préoccupation s'exprime de manière récurrente à partir d'avril 1966 dans une série d'articles du Bulletin d'information du CNC consacrée au «Cinéma spécialisé» (c'est-à-dire films produits et diffusés à des fins culturelles).

36 Décrets, Arrêtés et circulaires/ Ministère d'Etat chargé des Affaires culturelles, Arrêté du 07/ 10/1965, Journal officiel. Lois et décrets, I5/I0/1965, p. 9/23. 
plan agréé par le directeur général du CNC. Holleaux déclare à l'époque que l'opération «contribuera à doter cette institution d'un instrument de travail indispensable: le fichier central des films; elle permettra aussi utilisation plus efficace de ses richesses dont la préservation intégrale sera désormais méthodiquement organisée» ${ }^{37}$.

Les rapports entre la Cinémathèque française et l'Etat ne cesseront de se détériorer autour des travaux de la Commission (Mannoni, 2006 : 332-404; Louis, 2013: 494-530). La tentative, fort maladroite, de reprendre en main la gestion de la Cinémathèque française en remplaçant Langlois à la direction en février 1968 placera l'Etat dans l'obligation de créer son propre organisme de préservation des collections cinématographiques.

\section{- L'affaire Langlois et la création du Service des Archives du film du CNC}

L'affaire Langlois représente la collision de deux conceptions patrimoniales distinctes dans leurs modalités d'accomplissement, car il semble désormais admis que l'exposition des collections participe des pratiques patrimoniales. Celle de l'Etat, entend d'abord rationnaliser la gestion des collections. Celle de la Cinémathèque française, privilégie la dimension spectaculaire depuis sa fondation. En conclusion de l'épisode, le 23 avril 1968, le Ministère des Affaires culturelles diffuse un communiqué. Il reconnaît l'antériorité de l'initiative privée en matière de collecte mais pose, surtout, les bases d'une politique du patrimoine cinématographique à venir:

Le répertoire cinématographique pose le double problème de sa sauvegarde et de sa diffusion. [...]

La sauvegarde du patrimoine cinématographique demande des locaux aménagés pour la conservation des films, et des crédits pour les opérations de restauration. L'Etat, qui disposera prochainement de toutes les installations nécessaires, envisage de créer un organisme public qui aura vocation pour recevoir les dépôts de films et les conserver. Les conditions de ces dépôts feront l'objet de contrats entre les déposants, y compris la Cinémathèque française, et l'organisme public à la gestion duquel seront associés des représentants des professions cinématographiques et des déposants. La diffusion du répertoire cinématographique est actuellement assurée par des organismes privés, tels que les ciné-clubs ou certaines salles du circuit commercial, qui s'entendent avec les titulaires de droits d'exploitation pour projeter les films. Parmi eux, la Cinémathèque française occupe une place particulière. Aussi, pour lui permettre de poursuivre en toute liberté son rôle de diffusion, l'Etat est prêt à lui apporter une aide en nature en lui concédant l'utilisation de salles de projection dont il règlera les dépenses de fonctionnement. ${ }^{38}$

Priorité au film, pluralisme et complémentarité. Dans ce programme, l'Etat reconnaît la nécessité de la protection et de la projection, éludant le grand projet de musée qui était pourtant inscrit au Ve $\operatorname{Plan}^{39}$. En effet, en 1966, les

37 «Plan de conservation des films détenus par la Cinémathèque Française », Bulletin d'information du CNC, $n^{\circ} 103$, février 1967, p. 27.

38 «L'Etat définit 'officiellement' le rôle de la Cinémathèque », Le Monde, 23/04/1968.

39 Entre 1946 et 2006, la France définit, de manière indicative et incitative, des objectifs pour orienter les investissements dans les secteurs économiques prioritaires, sous l'égide du Commissariat au Plan, Le 
travaux du groupe cinéma de la commission de l'équipement culturel et du patrimoine artistique en vue du Ve Plan avaient abouti aux propositions budgétaires suivantes: Installation au Palais de Chaillot du Musée du cinéma (4 millions de francs), Construction du local de conservation des films à Bois d'Arcy (5 millions), tirage de copies de films menacés de destruction (3 millions), équipement d'un établissement de recherches (2 millions) (CNC, 1966: p.56). La création du Service des archives du film du CNC en 1969 marque finalement la mise en œuvre de ce projet non pas par un, mais par deux organismes distincts : les archives et la cinémathèque-musée. De plus, ce que l'Etat désigne désormais comme «patrimoine cinématographique» a évolué, n'incluant plus seulement les films produits avec son soutien mais, plus largement, ceux qui composent «le répertoire».

En 1968, la reconnaissance de l'utilité de l'exposition des films dans la construction patrimoniale constitue une réelle avancée, alors que la création d'un stock de films non lié à une cinémathèque marque quant à elle une réforme infrastructurelle importante. Dans ce double mouvement, les cinémathèques-musées exercèrent une influence déterminante.

Pour la période 1936-1968, la Cinémathèque française a occupé une place tout-à-fait centrale dans la formalisation et l'organisation du paysage institutionnel français, comme nous l'avons vu. En premier lieu: la Cinémathèque française a montré, grâce à sa contribution au débat public, que patrimoine, cinéma et musée constituaient les pôles interconnectés d'un nouvel espace du monde cinématographique, dédié au passé. En deuxième lieu, la Cinémathèque française a démontré au pouvoir politique que le processus patrimonial ne pouvait complètement s'accomplir sans intégrer des d'exposition des films. En cela, sa stratégie a consisté à inclure une monstration de type muséal dans le champ de compétences des cinémathèques qui préexistaient en France et à répondre ainsi aux attentes cinéphiliques de son époque. Elle a promu l'équivalence entre cinémathèque et musée, et ce modèle a été repris par d'autres cinéphiles qui ont implanté des organismes alternatifs sur le territoire. Cependant, cette appropriation par d'autres constitue aussi un geste critique qui signale la nécessité de rompre avec le système hyper centralisé que la Cinémathèque française a mis en place tout au long des années 1950. En troisième lieu, la détérioration de ses relations avec l'Etat la pose comme un contre-modèle et conduit à créer un organisme étatique spécifique pour la conservation du patrimoine cinématographique. Dès lors, la patrimonialisation du cinéma pourra s'émanciper d'un paradigme muséal jusqu'alors dominant.

A l'origine, en plus de tenter de rassembler une collection d'Etat, le Service des Archives du Film apporte un soutien aux projets développés localement, notamment quant à la conservation des éventuels fonds nitrate. En effet, le décret 69-675 relatif à la conservation des films par le CNC ne concerne que la conservation des films qui lui sont remis en dépôt ou acquis par ses soins. Seule l'activation du dépôt légal en 1977 rendra le bouleversement de paradigme de patrimonialisation réellement effectif. Là encore, la pression des cinémathèques, et des cinéphiles qui les animent, interviendra. Certaines soutiennent, comme celle de Toulouse, la publication dans Le Monde du 24 mars 1975 puis dans les colonnes de cinq revues spécialisées, un appel à la création d'une Cinémathèque

Cinquième Plan de développement économique et social se tient entre 1966 et 1970. 
nationale et à la création du dépôt légal des films (Marcorelles, 1975) ${ }^{40}$. Mais cette perspective ne fera cependant pas l'unanimité, ranimant les indéfectibles soutiens de la Cinémathèque française (Le Monde, 1975). Une première application du dépôt légal, qu'avait instauré la loi du 21 juin 1943, intervient avec un décret du 30 juillet 1975 mais ne concerne que les films autres que $35 \mathrm{~mm}$, entre autres œuvres audiovisuelles et multi-média ${ }^{41}$. Entre mai et juin $1977^{42}$, un décret d'application du dépôt légal spécifique aux films cinématographiques, sera publié. Désormais, le dispositif de patrimonialisation français articulera la collecte d'opportunité, menée essentiellement par les cinémathèques, et la collecte systématique, cadrée par la loi, des films et matériels publicitaires afférents $^{43}$. La loi du 20 juin 1992, rendue effective par le décret du 31 décembre $1993^{44}$ élargira le spectre des films concernés par le dépôt légal au CNC à la sauvegarde de tous les films - longs et courts métrages, français et étrangers, documentaires et fictions - ayant reçu un visa d'exploitation en France.

Bibliographie

AVRONSART. Sous le signe de Jean Epstein, Excelsior, Paris, 9 juin 1933 BORDE, Raymond. Les Cinémathèques. Lausanne : L'âge d'homme, 1983.

. Histoire de la Cinémathèque de Toulouse.Archives, 30/31, 1990.

; PERRIN, Charles. Les Offices du cinéma éducateur et la survivance du muet, 1925-1940. Lyon : Presses universitaires de Lyon, 2012.

CASANOVA. Paul Reboux nous dit ce que pourrait être le cinéma à l'Exposition Internationale de 1937. Paris-Soir, Paris, 23 fev. 1935

CENTRE NATIONAL DE CINEMATOGRAPHIE. Les Participations Financières de l'Etat dans la Production des Films Cinématographiques. Bulletin d'information. Paris, $n^{\circ}$ 8, mars-avril 1949, p. 2.

CENTRE NATIONAL DE CINEMATOGRAPHIE, Liste des films constituant le patrimoine cinématographique de l'Etat, 1965, np.

CENTRE NATIONAL DE CINEMATOGRAPHIE. an $^{\text {an }}$ de conservation des films détenus par la Cinémathèque Française», Bulletin d'information du CNC, $\mathrm{n}^{\circ} 103$, février 1967, p. 26.

CENTRE NATIONAL DE LA CINEMATOGRAPHIE FRANÇAISE. Bulletin d'information, $\mathrm{n}^{\circ} 98$, avril 1966, p.56

CHATAIGNER, Jean. Cinémathèque nationale. Le Journal, 19 jan. 1934. p.6.

COMITE D'HISTOIRE DU MINISTERE DE LA CULTURE. Malraux ministre au jour le jour. Souvenirs d'André Holleaux, conseiller d'Etat, directeur du cabinet d'André Malraux de 1962 à 1965. Paris: La Documentation française, 2004.

COMOEDIA, Paris, 27 nov. I913. p. 4

DANVERS, Guillaume. Une Exposition d'Art cinématographique organisée par

40 Les revues concernées sont : Les Cahiers du cinéma, Cinéma, Ecran 75, Positif, La Revue du Cinéma-Image et son.

4I En effet, le décret $n^{\circ} 75-696$ du 30 juillet 1975 fixant les conditions d'application aux œuvres audiovisuelles et multi-média de la loi du 21 juin 1943 relative au dépôt légal.

42 Le décret $n^{\circ} 77-535$ du 23 mai 1977 étant paru sans signature du Ministre, un décret rectificatif est publié au 15 juin sur conditions d'application aux films cinématographiques de la loi du 21 juin 1943, relative au dépôt légal.

43 Initialement prévue pour les films français, la collecte sera élargie par loi du 20 juin $1992\left(n^{\circ} 92-546\right)$ à l'ensemble des films ayant bénéficié d'un visa d'exploitation en France.

44 Loi $n^{\circ} 92-546$ du 20 juin 1992 relative au dépôt légal ; Décret $n^{\circ} 93-1429$ du 31 décembre 1993 relatif au dépôt légal. 
la Ville de Paris groupe les appareils des 'précurseurs' et les perfectionnements modernes. Comoedia, Paris, 23 mai I924. p.I

DARTOIS, Yves. La cinémathèque de la Ville de Paris. Comoedia, 16 mars 1928 , p.8.

DAVALLON, Jean. Le don du patrimoine: une approche communicationnelle de la patrimonialisation. Paris, Lavoisier, 2006.

DEGLESNE, Léo. Pour un Musée du Cinéma. La Griffe, 6 sept. 1936, p.8

DESLANDES, Richard. Histoire comparée du cinéma. II Du Cinématographe au cinéma, 1896-1906. Paris : Casterman, 1968.

DE BAECQUE, Antoine. La Cinéphilie. Invention d'un regard, histoire d'une culture. 1944- 1968. Paris: Fayard, 2003.

DE PASTRE, Béatrice. Créer des archives cinématographiques à Paris. L'oubli du père ou les héritiers parisiens de Boleslas Matuszewski. In : MAZARAKI, Magdalena (org.) Boleslas Matuszewski, Une nouvelle source de l'histoire. Magdalena Mazaraki, Paris :AFRHC/ Cinémathèque française, 2006.

D’HERBEUMONT, Louis. Bibliothèque et Musée du cinéma. Le Cinéopse, n ${ }^{\circ} 206$, OI oct 1936, p.I76

DUPIN, Christophe. Je t'aime moi non plus. Ernest Lindgren and Henri Langlois pioneers of the film archive movement. In : NOWELL-SMITH, Geoffrey; DUPIN, Christophe (org.) The British Film Institute, the government and film culture, 1933-2000. Manchester : Manchester University Press, 2012.

. First tango in Paris :The Birth of FIAF, 1936-1938. Journal of Film Preservation, 88, p.42-57, 2013.

. FIAF : From a Promising Start to Forced Hibernation ( 1938- 1945). Journal of Film Preservation, 99, p.76-89, 2018.

EMILE-BAYARD, Jean.A quand la création du Musée du cinéma français. Comoedia, Paris, 26 sept. 1936.

GAUTHIER, Christophe. La conservation et la consultation des films dans les années 1920 : l'exemple de la Cinémathèque Gaumont. Gazette des Archives, I73, p.225-230, 1996.

. La passion du cinéma. Cinéphiles, ciné-clubs et salles spécialisées à Paris de 1920 à 1928. Paris: École des chartes/Association française de recherche sur l'histoire du cinéma, 1999.

. Une Composition française. La mémoire du cinéma en France des origines à la seconde guerre mondiale. Thèse de doctorat soutenue à l'Université Paris I sous la direction de Pascal Ory : Paris, 2007.

GREMILLON, Jean. Le cinéma? Plus qu'un art! Ecrits et propos. 1925-1959. Pierre Lherminier, Paris: L'Harmattan, 2010.

HOMBOURGER, R. De 'Cœur fidèle' à 'l'Or des mers'. Comoedia, Paris, 03 juin 1933. p.6.

ICART, Roger. Abel Gance. Toulouse, CRDP : 1960.

JAMENSON, Amable. Les films patrimoine artistique. L'Ecran français, $n^{\circ} 5,0$ I août 1945. p. I2-I3.

JOCASTE. Le Matin, Paris, I 5 mai 1925. p.4

LA VIE des letres. Les Nouvelles littéraires, Paris, 27 mai 1933.

LANGLOIS, Henri. 'La Cinémathèque Française' en plein accord avec M. Jean-Emile Bayard, Comoedia, 0 I oct. 1936, p.4

. La Cinémathèque nationale. L’Education nationale. Décembre 
1950, $\mathrm{n}^{\circ}$ spécial: Bibliothèques, p. 44.

. Ecrits de cinéma (193/-1977). Bernard Benoliel et Bernard Eisens-

chitz (org). Paris: La Cinémathèque française/Flammarion, 2014.

LASPEYRES, Pierre-J. La Cinémathèque française en plein accord avec $M$. Jean-Emile Bayard. Comoedia, Paris, 0 I oct. 1936. p.4.

. Une Lettre de M. Gaston Thierry dit combien la réalisation est

ardemment souhaitée. Comoedia, Paris, 10 octobre 1936, p.4.

Le Petit Parisien, Paris, 27 nov. I913. p.7.

LEBRETON, R.L. Que mettrions-nous au musée du Cinéma? Comoedia, Paris, 3 jan. 1929. p.6.

LIAUSU, Jean-Pierre. Comoedia, 26 janvier 1936. p.6.

LOUIS, Stéphanie-Emmanuelle. Le Musée au pluriel. Faire voir le patrimoine cinématographique en France à l'heure de l'expansion cinéphile (1944-1968). Thèse de doctorat soutenue sous la direction de Christian Delage, EHESS, Paris, 2013.

. Le musée au pluriel. 1958-1968 : le patrimoine cinématographique au prisme de la décentralisation. In :VEZYROGLOU, Dimitri (org.) La Culture, une Affaire d'Etat (1945-1970). Paris, Comité d'histoire du ministère de la Culture, 2014.

. Un musée en suspens... L'implantation de Journées internationales du cinéma d'animation à Annecy et la question patrimoniale. In : DENIS, Sébastien ; DUCHET, Chantal ; MERIJEAU, Lucie ; PRUVOST-DELASPRE, Marie ; ROFFAT, Sébastien (org.) Archives et acteurs du cinéma des cinémas d'animation en France. Paris, L'Harmattan, 2014.

MANNONI, Laurent. Histoire de la Cinémathèque française. Paris : Gallimard, 2006. MARCORELLES, Louis. Cinq revues de cinéma demandent la création d'une cinémathèque nationale. Le Monde, Paris, 24 mars 1975.

. A propos de l'appel pour une cinémathèque nationale. Le Monde, Paris, 07 avril 1975.

OLMETA, Patrick La Cinémathèque française de 1936 à nos jours. Paris : CNRS éditions, 2000.

PETIT, Marcel. L'avenir du cinéma éducateur. L'œuvre, 29 jan. 1932. p.6.

POIRRIER, Philippe Les Politiques culturelles en France. Paris: La Documentation française, 2003.

PORNON, Charles. Esthétique du cinéma. Toulouse, CRDP : I96I.

. Dix mille scénarios en 27 stéréotypes. Toulouse, CRDP : 1964.

POULOT, Dominique. Bilan et perspectives pour une histoire culturelle des musées. Publics et Musées. 2, p. I25-I48, 1992.

SOUILLES-DEBATS, Léo. La Culture cinématographique du mouvement ciné-club. Une histoire de cinéphilies (1944-1999). Paris:AFRHC, 2017.

THEODORA. Le Matin, Paris, 03 nov. 1922. p.5

THIERRY, Gaston. Nous allons [avoir] un musée du cinéma! Paris-soir, Paris, 21 avril 1936. p.6.

Pour le Musée du Cinéma. Paris-soir, Paris, 12 mai 1936. p.8.

VERAY, Laurent. La représentation de la guerre dans les actualités françaises de 1914 à 19/8. In: 1895, revue d'histoire du cinéma, I7, p.3-52, 1994. 